\title{
Protein degradation in Parkinson disease revisited: it's complex
}

\author{
Han Li' and Ming Guo ${ }^{1,2}$ \\ 1UCLA ACCESS Graduate Program and 2Department of Neurology and Department of Molecular and Medical Pharmacology, Brain Research Institute, \\ David Geffen School of Medicine and Molecular Biology Institute, UCLA, Los Angeles, California, USA.
}

\begin{abstract}
Mutations in the genes PTEN-induced putative kinase 1 (PINK1), PARKIN, and $D J-1$ cause autosomal recessive forms of Parkinson disease (PD), and the Pink1/Parkin pathway regulates mitochondrial integrity and function. An important question is whether the proteins encoded by these genes function to regulate activities of other cellular compartments. A study in mice, reported by Xiong et al. in this issue of the JCI, demonstrates that Pink1, Parkin, and DJ-1 can form a complex in the cytoplasm, with Pink1 and DJ-1 promoting the $\mathrm{E} 3$ ubiquitin ligase activity of Parkin to degrade substrates via the proteasome (see the related article beginning on page 650). This protein complex in the cytosol may or may not be related to the role of these proteins in regulating mitochondrial function or oxidative stress in vivo.
\end{abstract}

Parkinson disease $(\mathrm{PD})$ is the second most common neurodegenerative disorder, and mutations in the genes PTEN-induced putative kinase 1 (PINK1, also known as Parkinson disease 6 [PARK6]), PARKIN (also known as $P A R K 2$ ), and $D J-1$ (also known as PARK7) cause autosomal recessive forms of $\mathrm{PD} /$ parkinsonism. PINK1 encodes a protein with a mitochondrial targeting sequence and a putative serine/threonine kinase domain, and PINK1 is predominantly localized to mitochondria (1). The Parkin protein contains two RING finger motifs, has E3 ubiquitin ligase activity in vitro, and is largely localized to the cytosol (1). The endogenous DJ-1 protein is found in mitochondria and cytosol, but the function of DJ-1 is not entirely clear (1). Studies on the functions of these genes may provide important insights into PD pathogenesis.

\section{Prior studies on protein degradation}

Most PD patients have intraneuronal inclusions in the form of ubiquitin-positive Lewy bodies and Lewy neurites. Given the presence of Parkin in Lewy bodies and the putative role of Parkin as an E3 ligase, much of the initial work on Parkin was focused

Conflict of interest: The authors have declared that no conflict of interest exists.

Nonstandard abbreviations used: PARK2, Parkinson disease 2; PD, Parkinson disease; PINK1, PTEN-induced putative kinase 1; PPD, Parkin/Pink1/DJ-1 (complex); Ub, ubiquitin polypeptide; UPS, ubiquitin-proteasome system.

Citation for this article: J. Clin. Invest. 119:442-444 (2009). doi:10.1172/JCI38619. on its potential role in regulating protein degradation via the ubiquitin-proteasome system (UPS). Ubiquitination is accomplished by covalently linking the ubiquitin polypeptide (Ub) to a lysine residue in a specific protein substrate and requires the sequential action of an E1 activating enzyme, an E2 conjugating enzyme, and an E3 ligase (2). E3 ubiquitin ligases can mediate monoubiquitination (the addition of a single Ub to the substrate protein), multiubiquitination (the addition of multiple single Ubs to different lysine residues in a target protein), and polyubiquitination (in which chains of four or more Ubs are formed by the linkage of $\mathrm{Ub}$ molecules to lysine residues in other Ub molecules) (2). These linkages are most often to lysine 48 (K48) or lysine 63 (K63) of the Ub polypeptide. Proteins to be degraded by the proteasome are largely K48 polyubiquitinated. In contrast, K63 polyubiquitination, as well as monoubiquitination and multiubiquitination, primarily function in non-degradative processes including signal transduction, transcriptional regulation, protein localization, and membrane trafficking (2).

Previous studies suggested that Parkin could function as an E3 ligase for proteasome-mediated protein degradation (3). A handful of substrates of Parkin, including Parkin itself and an $\alpha$-synuclein-interacting protein (Synphilin-1) (4), have been identified in vitro. If Parkin were indeed important in the degradative pathway in vivo, one would expect that the levels of its substrates should increase in Parkin- knockout mice. Unexpectedly, however, most of the substrates studied, including Synphilin-1, did not accumulate in Parkinnull mice (5). In addition, several studies have revealed that Parkin preferentially catalyzes monoubiquitination and K63-linked polyubiquitination of substrates including Synphilin-1 (6-8). These latter observations may offer potential explanations for the lack of substrate accumulation in vivo by implicating Parkin in a non-degradative, proteasome-independent process. Studies in Drosopbila and more recently in mammals have provided important insights into Parkin function, although whether Parkin possesses degradative or non-degradative functions remains to be determined.

\section{Central role of mitochondrial function in PD pathogenesis}

Flies lacking Parkin function show striking defects in mitochondrial morphology that are highly similar, if not identical, to those observed in Pink1 mutants (9-11). Genetic epistasis experiments have demonstrated that Parkin and Pink1 act in a common genetic pathway, with Pink1 positively regulating Parkin $(9,10)$. This Pink1/ Parkin pathway controls mitochondrial integrity at least in part via promotion of mitochondrial fission and/or inhibition of mitochondrial fusion (12-14). Consistent with these findings in Drosopbila, patients with PINK1 or PARKIN mutations have indistinguishable clinical features and also show mitochondrial defects $(1,15)$. Recent studies also suggest that Pink1 and Parkin regulate mitochondrial functions in mammals (16-19). These findings underscore the central importance of the Pink1/Parkin pathway in regulating mitochondrial integrity and function.

Parkin is localized largely in the cytosol, though it can be found within mitochondria or associated with the outer mitochondrial membrane in certain contexts (20-22). Meanwhile, Pink1 has been found within mitochondria in cells and in vivo (9, $23,24)$. The mechanism by which Parkin 
A

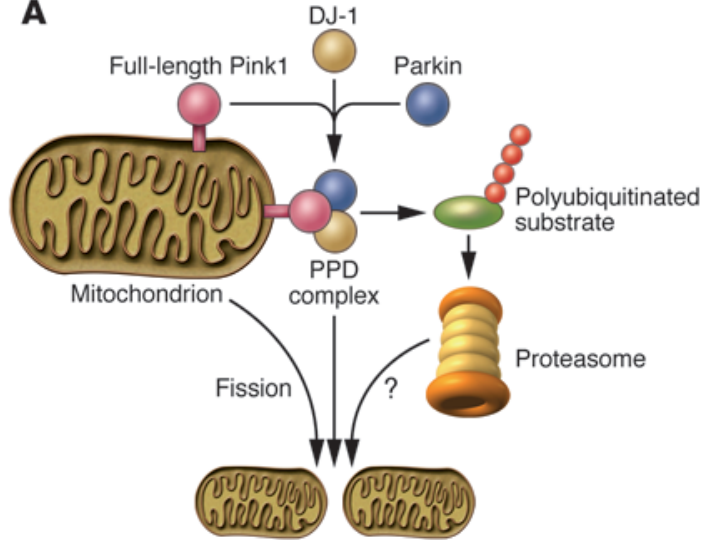

B

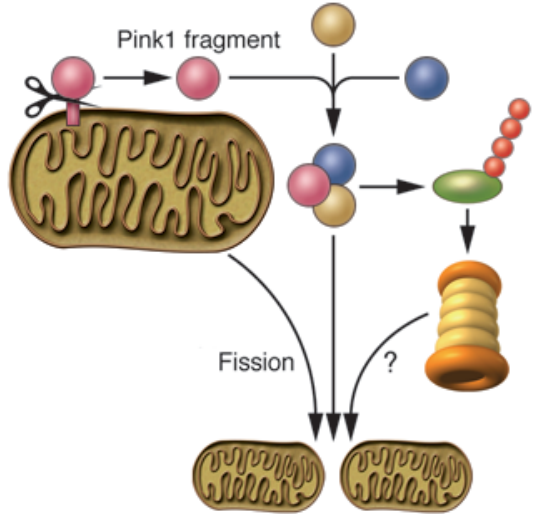

C

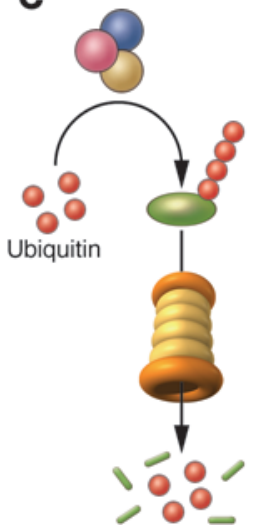

Figure 1

Three models for the role of the PPD complex. In this issue of the $\mathrm{JCl}$, Xiong et al. report that Pink1, Parkin, and DJ-1 bind to each other and form a PPD E3 ligase complex in which Pink1 and DJ-1 modulate Parkin-dependent ubiquitination and subsequent degradation of substrates via the proteasome (26). Previous work suggests that the Pink1/Parkin pathway regulates mitochondrial integrity and promotes mitochondrial fission in Drosophila (9-14). (A) Parkin and DJ-1 may be recruited to the mitochondrial outer membrane during stress (30, 31) and interact with Pink1. These interactions may facilitate the ligase activity of Parkin, thereby facilitating the turnover of molecules that regulate mitochondrial dynamics and mitophagy. The PPD complex may have other roles in the cytosol that result in degradative ubiquitination (26) and/or relay information from mitochondria to other cellular compartments. (B) Alternatively, Pink1 may be released from mitochondria after cleavage to interact with DJ-1 and Parkin in the cytosol. A and B differ in the site of action of the PPD complex and the cleavage status of Pink1. The complex forms on the mitochondrial outer membrane potentially containing full-length Pink1 in A, and in the cytosol with cleaved Pink1 in B. The question marks indicate speculative connections. Note that lack of $D J-1$ function results in phenotypes that are distinct from the mitochondrial phenotypes observed in null mutants of Pink1 or Parkin in Drosophila $(9,10,12,13,34)$. Thus, although the PPD complex is illustrated here as regulating mitochondrial fission, the role of $D J-1$ in vivo remains to be clarified. (C) It is also possible that the action occurs in the cytosol and is independent of the function of Pink1/Parkin in regulating mitochondrial integrity and function.

interacts with Pink 1 and acts on mitochondria has remained a mystery. Interestingly, a recent study on the topology of Pink1 suggests that Pink1 may be anchored in the mitochondrial outer membrane, with its kinase domain facing toward the cytoplasm (25), providing one possible route for the Pink1/Parkin interaction.

\section{Revisiting protein degradation in the cytosol}

In this issue of the JCI, Xiong and colleagues report a possible mechanism by which Parkin, Pink1, and DJ-1 might function outside of mitochondria in mice (26). Previous work had suggested that Pink1 binds to DJ1 (27), DJ-1 binds to Parkin (28), and Pink1 binds to Parkin $(1,29)$. Here, Xiong et al. extend these observations by showing that the 3 proteins co-fractionate in gel filtration assays and co-immunoprecipitation in vivo. These interactions were observed largely in the cytosol, rather than in the mitochondria. Moreover, when Pink1 or DJ-1 are overexpressed or provided directly in vitro, ubiquitination and degradation of Parkin substrates (Synphilin-1 and Parkin itself) via the UPS pathway were enhanced, while levels of ubiquitinated Parkin decreased in Pink1-mutant brain slice culture. The authors also show that pathogenic mutants of Pink1 and Parkin impaired the activity of the Parkin/Pink1/DJ-1 (PPD) complex, providing a potential mechanistic basis for how mutations in these proteins lead to $\mathrm{PD}$ pathogenesis. Based on these observations, the authors propose that Pink1, Parkin, and DJ-1 form a novel, cytoplasmically localized E3 ligase complex (the PPD complex) in which Pink1 and DJ-1 modulate Parkin-dependent ubiquitination and subsequent degradation of substrates. The authors also suggest that the PPD complex promotes the degradation of unfolded proteins. This conclusion is based largely on the observation that heat shock stress to cells in culture (which is presumed, though not shown, to cause misfolding of Parkin) resulted in increased ubiquitination and accumulation of Parkin, an effect that was suppressed when Pink1 was overexpressed. These results are intriguing, but heat shock is likely to alter the folding of many proteins and to affect the activity of myriad signaling pathways. Thus it is unclear whether the observed effects reflect direct interactions between Pink1, Parkin, and unfolded substrates. It will be particularly interesting to follow the fate of specific proteins known to be misfolded, when added to otherwise unperturbed cellular systems in which levels and activities of Pink1 and Parkin have been altered.

\section{Possible connections between mitochondrial dysfunction and protein degradation?}

How might the function of the PPD complex be related to the established roles of Pink1 and Parkin in regulating mitochondrial integrity and function? It is possible that the roles of Pink1/Parkin in regulating mitochondrial function are related to the potential degradative functions of the PPD complex (Figure 1, A and B). DJ-1 can be recruited to the mitochondrial outer membrane following oxidative stress (30). Upon mitochondrial uncoupling, overexpressed Parkin can also be selectively recruited onto impaired mitochondria, promoting their removal through autophagy (31). If the kinase domain of Pink1 does indeed face the cytoplasm (25), Parkin and DJ-1 might be recruited to the mitochondrial surface under certain conditions and may thereby interact with Pink1 at the mitochondrial outer membrane (Figure 1A). Perhaps this interaction facilitates the ubiquitin ligase activity of Parkin, thereby promoting the turnover of molecules that regulate mito- 
chondrial dynamics and mitophagy. A complication with this model, however, is that Xiong and colleagues report that while full-length Pink1 localized to mitochondria, a processed fragment of Pink1 was found predominantly in the cytoplasm, with this latter fragment being the version of Pink1 that predominantly interacts with Parkin (26). Similar cytosolic Pink1 fragments have been reported in earlier studies (32), suggesting a model in which Pink1 is cleaved and released from mitochondria under certain stress conditions. Cleaved forms of Pink1 may thus be able to interact with Parkin in the cytosol and thereby promote degradative ubiquitination or other cellular processes (Figure 1B). Pink1dependent activities in the cytoplasm may still serve to regulate mitochondrial function, for example by relaying information about mitochondrial status to other cellular compartments. The fact that a PD-causing mutation in the PINK1 kinase domain, G309D, compromises both the PPD complex function and mitochondrial integrity in Drosophila muscle and male germline (33) is consistent with the hypothesis that the PPD complex may regulate mitochondrial function. Alternatively, cytosolic Pink1 may carry out mitochondria-independent functions, perhaps also in complex with Parkin and DJ-1 (Figure 1C). It is important to note that loss of $D J-1$ results in phenotypes that are distinct from the mitochondrial defects associated with loss of Pink1/Parkin in Drosophila (9, 10, 34). If $\mathrm{DJ}-1$ is an obligatory component of this complex, these in vivo results would suggest that the PPD complex is not essential for regulation of mitochondrial integrity. Future experiments are needed to clarify the role of DJ-1 function.

\section{Summary}

In summary, while the current study by Xiong et al. (26) rekindles enthusiasm for exploring the roles of Parkin in mediating protein degradation via the UPS, it remains to be shown whether Parkin, in complex with Pink1 and DJ-1, carries out protein degradation in vivo. It will be particularly important to reconcile the observation by the authors that the PPD complex degrades Synphilin-1, with the in vivo observations demonstrating that Synphilin-1 levels do not accumulate in knockout mice lacking Parkin (5). Perhaps the PPD complex only functions to regulate Synphilin-1 levels when cells are stressed, but not under the conditions assayed in the Parkin-knockout mice. The Xiong et al. study offers an entry point for explorations of the role of Pink1, Parkin, and DJ-1 in the cytoplasm. The priority now becomes identifying in vivo contexts in which this complex functions.

\section{Acknowledgments}

We apologize to those whose work we were unable to cite because of space constraints. We thank Mark Dodson and Bruce Hay for helpful comments on the manuscript. This work is supported by funds from the McKnight Foundation, Alfred P. Sloan Foundation, Klingenstein Fellowship, American Parkinson Disease Association, and NIH (R01, P01, and K02 grants) to M. Guo.

Address correspondence to: Ming Guo, Department of Neurology, Department of Molecular and Medical Pharmacology, Brain Research Institute, UCLA David Geffen School of Medicine, 695 Charles Young Drive South, Los Angeles, California 90095, USA. Phone: (310) 206-9406; E-mail: mingfly@ucla.edu.

1. Dodson, M.W., and Guo, M. 2007. Pink1, Parkin, DJ-1 and mitochondrial dysfunction in Parkinson's disease. Curr. Opin. Neurobiol. 17:331-337.

2. Mukhopadhyay, D., and Riezman, H. 2007. Proteasome-independent functions of ubiquitin in endocytosis and signaling. Science. 315:201-215.

3. Kahle, P.J., and Haass, C. 2004. How does parkin ligate ubiquitin to Parkinson's disease? EMBO Rep. 5:681-685.

4. Chung, K.K., et al. 2001. Parkin ubiquitinates the alpha-synuclein-interacting protein, synphilin-1: implications for Lewy-body formation in Parkinson disease. Nat. Med. 7:1144-1150.

5. Ko, H.S., et al. 2005. Accumulation of the authentic parkin substrate aminoacyl-tRNA synthetase cofactor, p38/JTV-1, leads to catecholaminergic cell death. J. Neurosci. 25:7968-7978.

6. Lim, K.L., et al. 2005. Parkin mediates nonclassical, proteasomal-independent ubiquitination of synphilin-1: implications for Lewy body formation. J. Neurosci. 25:2002-2009.

7. Matsuda, N., et al. 2006. Diverse effects of pathogenic mutations of Parkin that catalyze multiple monoubiquitylation in vitro. J. Biol. Chem. 281:3204-3209.

8. Olzmann, J.A., et al. 2007. Parkin-mediated K63linked polyubiquitination targets misfolded DJ-1 to aggresomes via binding to HDAC6. J. Cell Biol. 178:1025-1038.

9. Clark, I.E., et al. 2006. Drosophila pink1 is required for mitochondrial function and interacts genetically with parkin. Nature. 441:1162-1166.

10. Park, J., et al. 2006. Mitochondrial dysfunction in Drosophila PINK1 mutants is complemented by parkin. Nature. 441:1157-1161.

11. Yang, Y., et al. 2006. Mitochondrial pathology and muscle and dopaminergic neuron degeneration caused by inactivation of Drosophila Pink1 is rescued by Parkin. Proc. Natl. Acad. Sci. U. S. A. 103:10793-10798.

12. Deng, H., Dodson, M.W., Huang, H., and Guo, M. 2008. The Parkinson's disease genes pink 1 and parkin promotes mitochondrial fission and/or inhibits mitochondrial fusion. Proc. Natl. Acad. Sci.U.S. A. 105:14503-14508.
13. Poole, A.C., et al. 2008. The PINK1/Parkin pathway regulates mitochondrial morphology. Proc. Natl. Acad. Sci. U. S. A. 105:1638-1643.

14. Yang, Y., et al. 2008. Pink1 regulates mitochondrial dynamics through interaction with the fission/fusion machinery. Proc. Natl. Acad. Sci. U. S. A. 105:7070-7075.

15. Ibanez, P., et al. 2006. Mutational analysis of the PINK1 gene in early-onset parkinsonism in Europe and North Africa. Brain. 129:686-694.

16. Exner, N., et al. 2007. Loss-of-function of human PINK1 results in mitochondrial pathology and can be rescued by parkin. J. Neurosci. 27:12413-12418.

17. Gautier, C.A., Kitada, T., and Shen, J. 2008. Loss of PINK1 causes mitochondrial functional defects and increased sensitivity to oxidative stress. Proc. Natl. Acad. Sci. U. S. A. 105:11364-11369.

18. Piccoli, C., et al. 2008. Mitochondrial respiratory dysfunction in familiar parkinsonism associated with PINK1 mutation. Neurochem. Res. 33:2565-2574.

19. Wood-Kaczmar, A., et al. 2008. PINK1 is necessary for long term survival and mitochondrial function in human dopaminergic neurons. PLOS ONE. 3:e2455.

20. Shimura, H., et al. 1999. Immunohistochemical and subcellular localization of Parkin protein: absence of protein in autosomal recessive juvenile parkinsonism patients. Ann. Neurol. 45:668-672.

21. Darios, F., et al. 2003. Parkin prevents mitochondrial swelling and cytochrome $c$ release in mitochondria-dependent cell death. Hum. Mol. Genet. 12:517-526.

22. Kuroda, Y., et al. 2006. Parkin enhances mitochondrial biogenesis in proliferating cells. Hum. Mol. Genet. 15:883-895.

23. Silvestri, L., et al. 2005. Mitochondrial import and enzymatic activity of PINK1 mutants associated to recessive parkinsonism. Hum. Mol. Genet. 14:3477-3492.

24. Gandhi, S., et al. 2006. PINK1 protein in normal human brain and Parkinson's disease. Brain. 129:1720-1731.

25. Zhou, C., et al. 2008. The kinase domain of mitochondrial PINK1 faces the cytoplasm. Proc. Natl. Acad. Sci. U. S. A. 105:12022-12027.

26. Xiong, H., et al. 2009. Parkin, PINK1, and DJ-1 form a ubiquitin E3 ligase complex promoting unfolded protein degradation. J. Clin. Invest. 119:650-660.

27. Tang, B., et al. 2006. Association of PINK1 and DJ-1 confers digenic inheritance of early-onset Parkinson's disease. Hum. Mol. Genet. 15:1816-1825.

28. Moore, D.J., et al. 2005. Association of DJ-1 and parkin mediated by pathogenic DJ-1 mutations and oxidative stress. Hum. Mol. Genet. 14:71-84.

29. Kim, Y., et al. 2008. PINK1 controls mitochondrial localization of Parkin through direct phosphorylation. Biochem. Biophys. Res. Commun. 377:975-980.

30. Canet-Aviles, R.M., et al. 2004. The Parkinson's disease protein DJ-1 is neuroprotective due to cysteine-sulfinic acid-driven mitochondrial localization. Proc. Natl. Acad. Sci. U. S. A. 101:9103-9108.

31. Narendra, D., Tanaka, A., Suen, D.F., and Youle, R.J. 2008. Parkin is recruited selectively to impaired mitochondria and promotes their autophagy. J. Cell Biol. 183:795-803.

32. Beilina, A., et al. 2005. Mutations in PTEN-induced putative kinase 1 associated with recessive parkinsonism have differential effects on protein stability. Proc. Natl. Acad. Sci. U. S. A. 102:5703-5708.

33. Yun, J., et al. 2008. Loss-of-function analysis suggests that Omi/HtrA2 is not an essential component of the PINK1/PARKIN pathway in vivo. J. Neurosci. 28:14500-14510.

34. Meulener, M., et al. 2005. Drosophila DJ-1 mutants are selectively sensitive to environmental toxins associated with Parkinson's disease. Curr. Biol. 15:1572-1577. 


\title{
Shock the heat shock network
}

\author{
Çiğdem Atay, Serkan Uğurlu, and Nesrin Özören \\ Department of Molecular Biology and Genetics, Apoptosis and Cancer Immunology Laboratory (AKIL), Boğaziçi University, Istanbul, Turkey.
}

\begin{abstract}
The targeting of tumors is made possible through establishing protein signatures specific for each cancer type. The recent recognition of the higher expression levels of HSP90 and its accumulation in tumor cell mitochondria has made the HSP90 network a feasible target for neutralization. HSP90 antagonizes the mitochondrial permeability transition, blocking cytochrome $c$ release and apoptosis. In this issue of the JCI, Kang et al. report the synthesis of Gamitrinibs, which target mitochondrially localized HSP90, specifically killing human cancer cell lines, and provide a fresh approach for cancer treatment (see the related article beginning on page 454 ).
\end{abstract}

The speed of accumulation of experimental data about normal as well as cancer cells has increased exponentially in the last several decades. Presently, our arsenal of knowledge is equipped with detailed information about cell proliferation, differentiation, and cell death induction pathways and the myriad of intricate interactions among them. Abnormalities in tumor suppressor genes and oncogenes have been correlated with disease states, and all of these tremendous advances have resulted in the heightened expectation that novel and better cancer therapies are clearly within reach. Yet, the most frequently applied treatment strategies continue to rely on "old school" therapies, combining surgery, chemotherapy, and/or radiotherapy. Chemotherapy and radiotherapy target proliferating cells, which include the rapidly dividing tumor cells but do not exclude normally proliferating cells of the skin and gastrointestinal tract. The generally low efficiency of cures for advanced cancers, the severe side effects of current therapy regimens, and the risk of posttherapeutic relapse have all contributed to the current and ongoing rush to find novel alternative therapeutic approaches (1).

\section{New generation of anticancer drugs}

Ideally, 21st century, clever, anticancer drugs are expected to target tumor cells

Authorship note: Çiğdem Atay and Serkan Uğurlu contributed equally to this work.

Conflict of interest: The authors have declared that no conflict of interest exists.

Nonstandard abbreviations used: $17-A A G, 17-$-allylamino)-17-demethoxygeldanamycin; $C Y P D$, cyclophilin D; GA, geldanamycin; Gamitrinib, GA mitochondrial matrix inhibitor.

Citation for this article: J. Clin. Invest. 119:445-447 (2009). doi:10.1172/JCI38681. specifically and spare damage to normal tissues. Thus, the search for tumor-specific markers or signatures has become the major focus of genomics, proteomics, and systems biology studies (2). It has been easier to find the signatures of certain types of cancers; for example, the human ERBB2/ neu (HER2/neu) protein is overexpressed in $30 \%$ of breast cancer patients (3) and the identification of this marker has made possible the generation of a neutralizing HER2/neu monoclonal antibody (known as Herceptin or trastuzumab), currently used successfully in the clinic (4). Trastuzumab is a breakthrough in the fight against cancer and provides the impetus for other researchers in their studies.

Another molecular signature, the overexpression of antiapoptotic BCL2 family members in human leukemias $(5,6)$, melanoma (7), and hepatocellular carcinoma (8), was used to generate a distinct class of molecular drugs. In this case, the BH3 domain of proapoptotic BCL2 family members or synthetic drugs mimicking the $\mathrm{BH} 3$ domain were used to neutralize the apoptosis-blocking action of BCLXL, BCLW, and/or BCL2 $(9,10)$. The aim was to tip the balance of expression of antiapoptotic/proapoptotic BCL2 family members in order to induce cell death. The most successful $\mathrm{BH} 3$ mimetic in phase III trials is ABT-737, which is currently used to treat primary chronic lym-

The mechanism of action of the drugs discussed above is based on targeting singular protein products, and the success of these drugs is exceptional considering the many thousands of compounds that have been tested in clinical trials and have failed. Drug designers have now begun to focus phocytic leukemia $(11,12)$. on identifying drugs that target signaling pathways, rather than singular proteins. Yet, another aspect of pathway-oriented drug discovery concerns the compartmental distribution of the components of the pathway at hand. The targeting of nodal signaling proteins localized in specific subcellular organelles, without affecting the expression or activities of these proteins in other cellular compartments, opens a new window for designing more effective anticancer drugs.

\section{HSP90 network activity in tumor cell mitochondria}

In this issue of the JCI, Kang et al. provide evidence of the successful utilization of a quite recently identified tumor signature, the mitochondrial accumulation of HSP90-network proteins, for apoptosis induction (13). Using Western blot analysis, mitochondrial HSP90 was previously found to be constitutively expressed at high levels in cervical carcinoma (HeLa), breast cancer (MCF-7), colon cancer (HCT-116), and B cell lymphoma (Raji) cell lines, suggesting that HSP90 may be critically important for tumor cell growth and/or survival (14). The same group of investigators had already shown via immunohistochemistry that mitochondria of tumor cells, but not most normal tissue samples, contain HSP90 and its related molecule TNF receptor-associated protein 1 (TRAP-1) (14). HSP90 and TRAP-1 were determined to interact with cyclophilin D (CYPD) and block its ability to cause mitochondrial outer membrane permeabilization, which is considered to be responsible for engaging the apoptotic cascade in numerous cell death pathways (Figure 1) $(14,15)$.

Normally, HSPs are upregulated upon establishment of stressful conditions, such as hyperthermia, oxidative damage, lack of nutrients, and others, and their main function is to serve as chaperones and catalyze the proper folding of certain client proteins (16). HSPs have been shown to regulate apoptosis signaling pathways at several steps. For example, HSP70 binds to the apoptosome component APAF1 and negatively regulates the 